\title{
Fabrication and Characterization of Highly Oriented N-Doped ZnO Nanorods by Selective Area Epitaxy
}

\author{
Yang Zhang, ${ }^{1}$ Shulin Gu, ${ }^{1}$ Kun Tang, ${ }^{1}$ Jiandong Ye, ${ }^{1}$ Haixiong Ge, ${ }^{2}$ Zhengrong Yao, \\ Shunming Zhu, ${ }^{1}$ and Youdou Zheng ${ }^{1}$ \\ ${ }^{1}$ National Laboratory of Solid State Microstructures, School of Electronic Science \& Engineering, Nanjing University, \\ Nanjing 210046, China \\ ${ }^{2}$ National Laboratory of Solid State Microstructures, Department of Materials Science and Engineering, \\ College of Engineering and Applied Sciences, Nanjing University, Nanjing 210093, China
}

Correspondence should be addressed to Shulin Gu; slgu@nju.edu.cn and Kun Tang; ktang@nju.edu.cn

Received 28 February 2015; Accepted 15 April 2015

Academic Editor: Meiyong Liao

Copyright (C) 2015 Yang Zhang et al. This is an open access article distributed under the Creative Commons Attribution License, which permits unrestricted use, distribution, and reproduction in any medium, provided the original work is properly cited.

\begin{abstract}
High-quality nitrogen-doped $\mathrm{ZnO}$ nanorods have been selectively grown on patterned and bare $\mathrm{ZnO}$ templates by the combination of nanoimprint lithography and chemical vapor transport methods. The grown nanorods exhibited uniformity in size and orientation as well as controllable density and surface-to-volume ratio. The structural and optical properties of $\mathrm{ZnO}$ nanorods and the behaviour of $\mathrm{N}$ dopants have been investigated by means of the scanning electron microscope, photoluminescence (PL) spectra, and Raman scattering spectra. The additional vibration modes observed in Raman spectra of N-doped ZnO nanorods provided solid evidence of $\mathrm{N}$ incorporation in $\mathrm{ZnO}$ nanorods. The difference of excitonic emissions from $\mathrm{ZnO}$ nanorods with varied density and surface-to-volume ratio suggested the different spatial distribution of intrinsic defects. It was found that the defects giving rise to acceptor-bound exciton $\left(\mathrm{A}^{0} \mathrm{X}\right)$ emission were most likely to distribute in the sidewall surface with nonpolar characteristics, while the donor bound exciton $\left(\mathrm{D}^{0} \mathrm{X}\right)$ emission related defects distributed uniformly in the near top polar surface.
\end{abstract}

\section{Introduction}

As a direct bandgap semiconductor, with its bandgap of $3.37 \mathrm{eV}$ and binding energy of $60 \mathrm{meV}$ at room temperature, zinc oxide $(\mathrm{ZnO})$ materials in forms of bulk, films, and nanostructures have shown versatile potential applications in electronic and optoelectronic devices, such as sensors $[1,2]$, light-emitting diodes [3, 4], and solar cells [5]. $\mathrm{ZnO}$ nanorods have attracted much attention due to the easy fabrication procedure via various growth techniques including metalorganic chemical vapor deposition (MOCVD) [6], thermal evaporation [7], vapor liquid solid deposition [8,9], and pulsed laser deposition (PLD) [10]. Besides, the selective area epitaxy of well-aligned high quality $\mathrm{ZnO}$ nanostructures has also been achieved [11-13]. In micron scale, conventional photolithography is easy and widely used to achieve the required patterns on the substrates [11], but as the dimension is shrunk into nanometer scale, electron-beam lithography
(EBL) $[12,13]$ is mostly used. However, EBL is relatively expensive and not suitable for practical application. Alternatively, nanoimprint lithography method $[14,15]$ provides an available and inexpensive choice for nanoscaled patterning in nanostructures fabrication.

The fabricated $\mathrm{ZnO}$ nanorods are usually oriented along $c$-axis and show weak dependence on the employed substrates. The top surface of $\mathrm{ZnO}$ nanorods is in either (0001) or $(000 \overline{1})$ orientation, exhibiting polar nature with $\mathrm{Zn}$ or O termination, respectively. A nonpolar (10 $\overline{1} 0)$ surface has the smallest surface formation energy among low index surfaces, and thus, $(10 \overline{1} 0)$ facets would be the major constituting facet of a crystalline $\mathrm{ZnO}$ nanomaterial [16]. In fact, the sidewall surface of $\mathrm{ZnO}$ nanorods is usually in the $(10 \overline{10})$ direction, which is nonpolar with equal numbers of $\mathrm{Zn}$ and $\mathrm{O}$ atoms on the surface. Nanometer-sized materials have a high surface-to-volume ratio, which critically affects the electronic and optical properties. In particular, when the $\mathrm{ZnO}$ nanorods 
have smaller diameters, their surface-to-volume ratios are higher and the surface effect is expected to be more distinguished. The surface effect should have a close correlation with the large density of dangling bonds existing in the sidewall surface of $\mathrm{ZnO}$ nanostructures, which have been ascribed to be the intrinsic defects, such as oxygen or zinc vacancies $\left(V_{O}\right.$ or $\left.V_{Z n}\right)$, and have a great effect on the optical properties of the nanorods.

Photoluminescence has been proven to be a powerful tool to study the optical properties of $\mathrm{ZnO}$ nanorods. For wide bandgap semiconductors, such as $\mathrm{GaN}$ and $\mathrm{ZnO}$, He$\mathrm{Cd}$ laser at the wavelength of $325 \mathrm{~nm}$ has been used as the excitation source, which has a small penetration depth of only $60 \mathrm{~nm}$ in $\mathrm{ZnO}$ material. Thus, the observed photoluminescence in $\mathrm{ZnO}$ films is mostly contributed from the region near surface, even taking the contribution of the recombination from the carriers located within the diffusion length of the excited region into account. Therefore, the overall photoluminescence spectra of $\mathrm{ZnO}$ nanorods can be regarded as the superposed radiative recombination signals mostly from both the top surface and the sidewall surface. The typical observed complicated $\mathrm{ZnO}$ luminescence spectra are then significantly influenced by the crystal morphology, thus leading to the divergence of optical emissions reported on various $\mathrm{ZnO}$ nanorods and exhibiting strong dependence on the sizes, morphologies, and growth techniques of $\mathrm{ZnO}$ nanostructures.

In fact, the optical properties of different facet $\mathrm{ZnO}$ film for both polar and nonpolar surfaces have been investigated. Different polarity surfaces have been found to lead different emission properties, including the kind of the emission and its intensity [17, 18]. For instance, the luminescent properties of the $\mathrm{Zn}$-polar and O-polar faces have been reported to be extremely different: the PL intensity of the $\mathrm{Zn}$-polar face is stronger than that of the O-polar face and the PL intensity of the zero-phonon free excitons relative to that of phonon replicas is stronger in the $\mathrm{O}$-polar face than that in the $\mathrm{Zn}$-polar face. The origin of the observed differences in PL properties has been discussed in terms of the difference of exciton-phonon coupling strengths, opposite band bending effect, and difference of the adsorption [17]. The opposite band bending effects at the two polar faces have been suggested to reduce the absorption effect at the O-polar face compared to the Zn-polar face [19]. As for nanomaterials, the similar differences observed in the CL spectra of O- (FX dominate) and $\mathrm{Zn}$ (DX dominate)-polar $\mathrm{ZnO}$ nanorods can be directly correlated with the distinct photoluminescence properties in $\mathrm{O}$ - and $\mathrm{Zn}$-polar $\mathrm{ZnO}$ epilayers and single crystals. It was found that the incorporation of impurities both in terms of concentration and nature may also depend on the polarity of $\mathrm{ZnO}$ NWs [20].

Moreover, the high surface-to-bulk ratio and the sidewall nonpolar surface existing in the nanorods may cause the observed emissions to be much more complicated. For nanorods array, the surface will include both the top surface and the sidewall surface, which should have important but different contribution to the observed emissions. Photoluminescence measurement can hardly get detailed information about such spatial distribution of the luminescence nor related defects on the surface of $\mathrm{ZnO}$ nanorods.. The different facets in $\mathrm{ZnO}$ nanorods surface even aggravate the complication of observed photoluminescence. In contrast to photoluminescence, cathodoluminescence can be easily applied to distinguish the observed emissions from the nanorods and correlate them with the nanorod structures. It is reported that the observed visible emission, which can be related with the existing surface defects, such as zinc or oxygen vacancies [21, 22], comes from the sidewall surface of nanorods. The band edge emission is believed mostly to come from the interior region in the nanorods. This indicates that the light emission spectra of $\mathrm{ZnO}$ observed from diverse morphology cannot be directly compared, although some common spectral features are present [23].

Besides the relationship between the existing intrinsic defects and the visible green band emission, the spatial distribution of near band edge emission in $\mathrm{ZnO}$ nanorods has also been deeply investigated via photoluminescence. Such inhomogeneity in optical properties of $\mathrm{ZnO}$ nanorods has actually been observed via the angular dependent PL measurements. The luminescence detected from the nanorods illuminated with the laser beam parallel to their growth axes shows that the near band emission (NBE) is dominated by the A-line and its LO phonon replicas while the emission collected from the nanorods illuminated from their lateral side exhibits higher contribution of free exciton [24]. The study suggests that $\mathrm{Zn}$ interstitials or some other donors are less formed in the sidewall surface, leading to a low carrier concentration within nonpolar facet. However, the investigation of detailed distribution of the above intrinsic defects in the $\mathrm{ZnO}$ nanorods is still challenging.

In this paper, the $\mathrm{ZnO}$ nanorods doped by nitrogen with different surface-to-bulk ratio have been fabricated on patterned and bar $\mathrm{ZnO}$ films, respectively, by a selective area chemical vapor transport (CVT) growth method. The growth mechanism of the $\mathrm{ZnO}$ nanorods on $\mathrm{SiO}_{2}$ patterned and nonpatterned $\mathrm{ZnO}$ seed films has been proposed. The correlation of optical emission and defects formation in topmost surface and sidewall surface has been established via surface morphological observation, Raman scattering, and low-temperature photoluminescence spectra. Distinctive shallow acceptors are observed to be preferentially formed on the sidewalls of the nanorods. Concerning the p-type doping in $\mathrm{ZnO}$ films which is still challenging, our study paves a way to realize economic and mass-productive controllable growth of well-patterned $\mathrm{ZnO}$ nanorods and to enhance the acceptor incorporation efficiency in low-dimension $\mathrm{ZnO}$ nanostructures grown at a high substrate temperature.

\section{Experimental Details}

In order to thoroughly control the uniformity of the crystal orientation and polarity of the nucleation surface, thick $\mathrm{ZnO}$ films grown by two-step growth procedure via metal-organic chemical vapor deposition have been used as seed layers for the growth of $\mathrm{ZnO}$ nanorods. The seed layer exhibited uniform Zn-polarity and high crystalline quality, as reported elsewhere [25]. A thin $\mathrm{SiO}_{2}$ barrier layer at a thickness of $60 \mathrm{~nm}$ as the selective mask was then deposited on 
the as-grown $\mathrm{ZnO}$ seed layer by plasma enhanced chemical vapor deposition (PECVD). Then a layer of hot embossing resist material was spin-coated on the $\mathrm{SiO}_{2} / \mathrm{ZnO} /$ sapphire substrates. A module of arrayed circular cylinder $(200 \mathrm{~nm}$ in diameter and $90 \mathrm{~nm}$ in height) of $400 \mathrm{~nm}$ period was prepared. The subsequent process was the nanoimprint lithography, and followed by this process, the hot embossing resist material was solidified at a higher temperature. The resisting material $/ \mathrm{SiO}_{2} / \mathrm{ZnO} /$ sapphire substrates were then etched by reactive ion etching process and the rest resisting material was lifted off by dimethylbenzene in ultrasonic cleaner. Thus, diameter and period of the hole can therefore be patterned on $\mathrm{SiO}_{2} / \mathrm{ZnO} /$ sapphire substrates at the dimension of $200 \mathrm{~nm}$ and the period of $400 \mathrm{~nm}$.

The growth of vertically aligned $\mathrm{ZnO}$ nanorods was carried out in a horizontal tube furnace via the chemical vapor transport method without employing any catalysts. The source material was a mixture of high purity $\mathrm{ZnO}$ and graphite powders ( $\mathrm{ZnO}: \mathrm{C}=1: 1$ by weight), which was placed in the centre of the quartz tube put in a horizontal furnace operated at the temperature of $950^{\circ} \mathrm{C}$. The substrate placed downstream was the prepared $\mathrm{ZnO}$ template on sapphire at the thickness of $2 \mu \mathrm{m}$ grown by the MOCVD method as seed layers. For $\mathrm{ZnO}$ nanorods growth, $\mathrm{N}_{2}$ was used as the carrier gas, while $\mathrm{N}_{2} \mathrm{O}$ was of both $\mathrm{O}$ and $\mathrm{N}$ sources. The flow rates of $\mathrm{N}_{2}$ and $\mathrm{N}_{2} \mathrm{O}$ were $100 \mathrm{sccm}$ and $1 \mathrm{sccm}$, respectively. A radiofrequency plasma generator was used for the ionization of $\mathrm{N}_{2} \mathrm{O}$ to produce both efficient $\mathrm{N}$ and $\mathrm{O}$ precursors for growth.

To get different intensity and surface-to-volume ratio of $\mathrm{ZnO}$ nanorods, three different kinds of samples have been grown. Sample A was grown on the patterned substrate for 20 minutes. Sample B was grown with the same condition but long growth time of $60 \mathrm{~min}$. Sample $\mathrm{C}$ was also grown for one hour but directly on $\mathrm{ZnO}$ substrate.

The morphology of the as-grown $\mathrm{ZnO}$ nanorods was characterized by a 5350 NE Dawson Creek Derive scanning electron microscopy (SEM) attached with energy dispersive analyser. The crystalline quality of all samples was analyzed by X-ray diffraction (XRD) preformed on a Rigaku D/MAX2500 using $\mathrm{Cu} \mathrm{K} \alpha$ radiation at $40 \mathrm{kV}$ and $200 \mathrm{~mA}$ by step scanning with a step size of $0.02^{\circ}$. The $514 \mathrm{~nm} \mathrm{Ar+} \mathrm{laser}$ with a power of $5 \mathrm{~mW}$ was used as the excitation source in micro-Raman spectroscopy at RT and the measurement was performed under a backscattering configuration. Photoluminescence spectra were recorded in the temperature of $9 \mathrm{~K}$, excited by a He-Cd laser with a wavelength of $325 \mathrm{~nm}$. The laser beam was impinged on the sample surface with an angle of approximately $60^{\circ}$. The excited PL emission was measured with a JY-Horiba monochromator, aligned normal to the sample surface. The chemical configuration of the elements was determined by X-ray photoelectron spectrometry with an $\mathrm{Al} \mathrm{K} \alpha \mathrm{X}$-ray monochromatic source at $1486.6 \mathrm{eV}$ (Thermo Fisher Scientific Inc., Model K-Alpha).

\section{Results and Discussions}

Figure 1 shows the scanning electron microscope images of $\mathrm{ZnO}$ nanorods grown in different conditions. From sample
A (Figure 1(a)), it can be clearly seen that $\mathrm{ZnO}$ nanorods were achieved only in the area of exposed $\mathrm{ZnO}$ layers, while on $\mathrm{SiO}_{2}$ layers no growth occurred, which shows that $\mathrm{ZnO}$ nanorods grew selectively on $\mathrm{ZnO}$ and $\mathrm{SiO}_{2}$ substrates. It can also be seen that $\mathrm{ZnO}$ nanorods have different growth density and size distribution on both substrates. On bare $\mathrm{ZnO}$ substrates, $\mathrm{ZnO}$ nanorods have a dense distribution, the diameter of which mostly ranges from $150 \mathrm{~nm}$ to $400 \mathrm{~nm}$ (Figure 1(f)). While on patterned $\mathrm{ZnO}$ substrates, $\mathrm{ZnO}$ nanorods of sample B were evenly distributed and the size of them was concentrated at $720 \mathrm{~nm}$ (Figure 1(e)). Consequently, we can conclude that, on patterned $\mathrm{ZnO}$ substrates, the growth of $\mathrm{ZnO}$ nanorods can be precisely controlled and we can get good $\mathrm{ZnO}$ nanorod arrays.

It is noted that the density of $\mathrm{ZnO}$ nanorods grown on sample B (Figure 1(b)) is about one-fifth that obtained on sample C (Figure $1(\mathrm{c})$ ), but the averaged size is about 3 times larger than that of sample C. As shown in Figure 2, for the above $\mathrm{ZnO}$ nanorods growth on barely or patterned $\mathrm{ZnO}$ film, the density and size of $\mathrm{ZnO}$ nanorods are finally determined by the formation of the initial nucleus on the substrate. For bare $\mathrm{ZnO}$ film (Figure 2(b)), the whole surface is available for the formation of the nucleation sites for $\mathrm{ZnO}$ nanorods growth (Figure 2(b)(1)), so the size of the nucleus will be decided by the unoccupied space between the neighbored nuclei, which determine the amount of adsorbed radicals which may be possible to migrate into the nucleus and so contribute to the growth of the initial nucleus. For the growth conditions as employed in the work, almost the whole surface will be covered by the grown $\mathrm{ZnO}$ nanorods (Figure 2(b)(2)). For patterned $\mathrm{ZnO}$ film in the work (Figure 2(a)), only partial $\mathrm{ZnO}$ surface is exposed to the ambient growth (Figure 2(a)(1)). However, the adsorption reaction radicals on the covered $\mathrm{SiO}_{2}$ surface have a high migration rate and can also move and incorporate with the nucleation sites (Figure 2(a)(2)), resulting in a much larger nucleus (Figure 2(a)(3)). In this case, much larger sizes of $\mathrm{ZnO}$ should be formed on sample B. According to the scanning electron microscopy image, we get the distribution of the $\mathrm{ZnO}$ nanorods size and number for both cases, which agree well with the above suggestion and discussion.

The XRD spectra of samples $\mathrm{A}, \mathrm{B}$, and $\mathrm{C}$ are shown in Figure 3. The inset shows the $\mathrm{ZnO}$ (0002) rocking curve (XRC) for all the samples. From the $\omega-2 \theta$ patterns, it can be seen that only the $\mathrm{ZnO}$ (0002) diffraction peaks have been detected, indicative of the desired $c$-axis preferential growth for the $\mathrm{ZnO}$ nanorods. Furthermore, the intensity of the (0002) peak drops for sample C, and the width of the $\omega$ rocking curves increases from 297 arc sec (sample A) and 276 arc sec (sample B) to $423 \mathrm{arc} \mathrm{sec}$ (sample C). The decreased XRD intensity and the increased XRC width for sample $\mathrm{C}$ have been ascribed to the relatively degraded crystalline quality of the grown nanorods as compared to the $\mathrm{ZnO}$ seed film, since the grown nanorods are much denser and will contribute more to the detected XRD patterns.

For photoluminescence measurements on $\mathrm{ZnO}$ nanorods array, the space between the neighbored nanorods will have a great effect. Small space like the case of nanorods grown on sample $\mathrm{C}$ should give a little contribution from the sidewall 


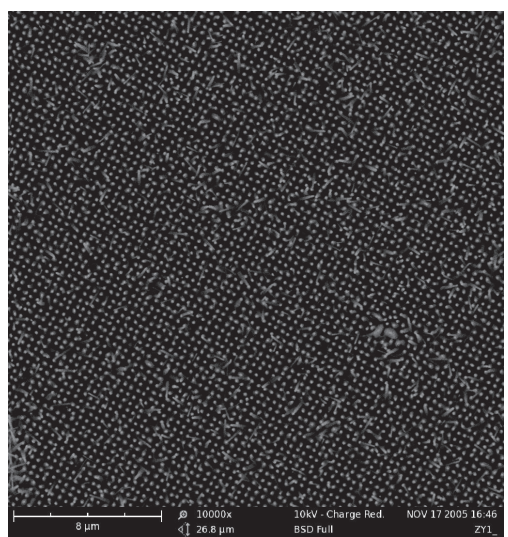

(a)

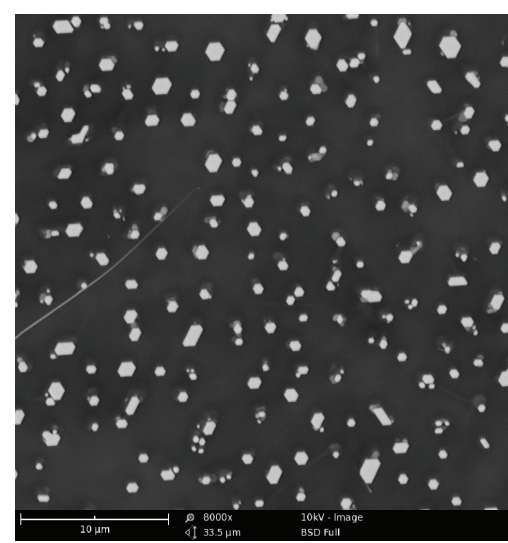

(b)

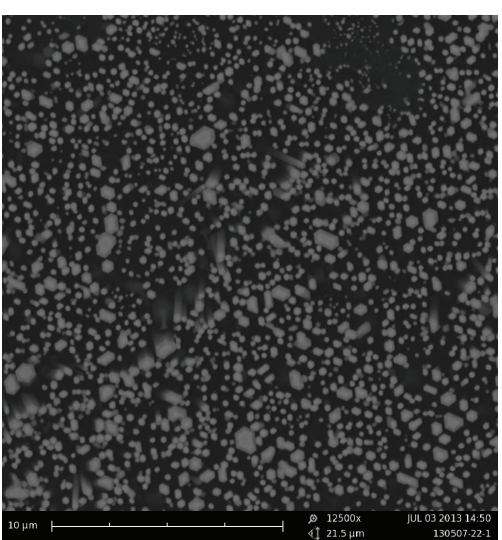

(c)

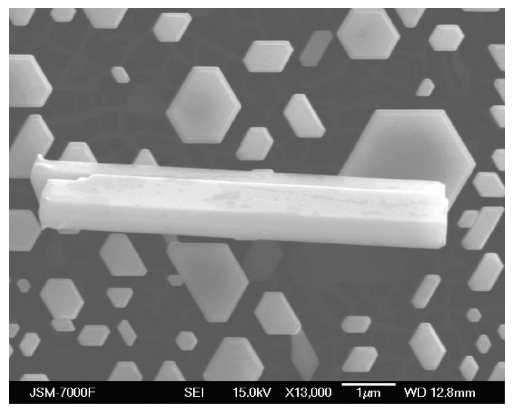

(d)

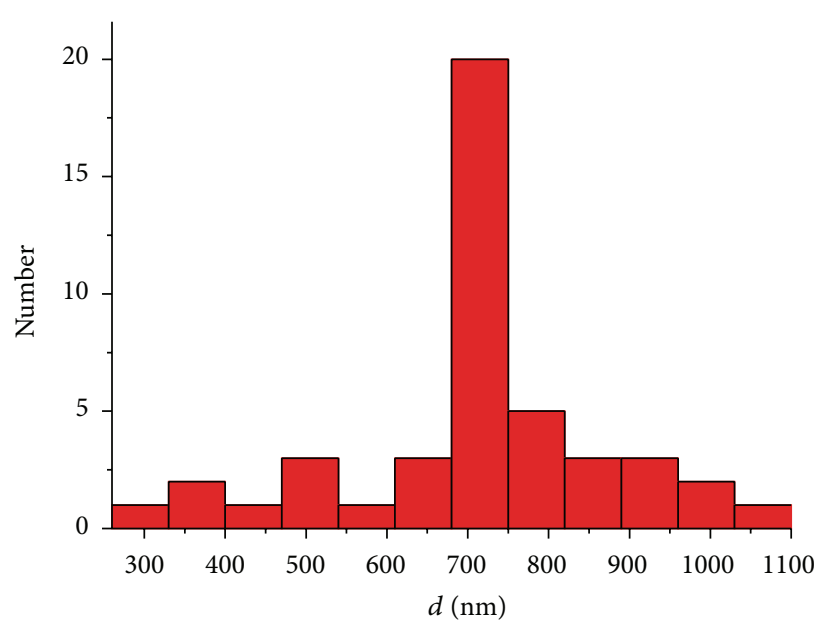

(e)

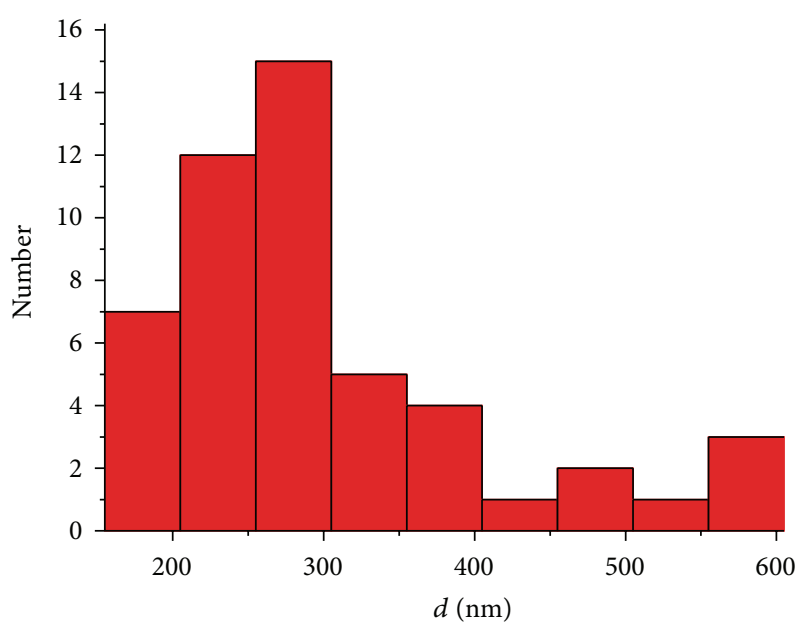

(f)

Figure 1: SEM images ( $a, b, c)$ of $\mathrm{ZnO}$ nanorods grown on patterned (a: sample A and b: sample B) and bare (c: sample C) ZnO substrates. (d) An amplified image of the $\mathrm{ZnO}$ nanorods, showing dense hexagonal nanorods in the size of tens to hundreds of nanometers. (e) and (f) The plot of the nanorod number versus the nanorod size distribution (e: sample B, f: sample C).

surface to the final emission, due to limited penetration depth of the incident laser and also less collection of the emission from the sidewall surface. However, a large space similar to the case on sample B should certainly give a high contribution from the sidewall surface. Considering the fact that the top surface coverage is lower than that of sample C, the contribution from the top surface for sample B might be weaker than the case for sample C. Therefore, the emission intensity ratio for the contribution from the top surface over the sidewall surface can be moderated and the different contribution may be evaluated in the work.

Figure 4 shows the photoluminescence (PL) spectra of samples $\mathrm{A}, \mathrm{B}$, and $\mathrm{C}$ measured at $9 \mathrm{~K}$. The features of $\mathrm{PL}$ spectra can be classified into two categories: near band emission (around $3.3 \mathrm{eV}$ ) and deep band emission (around $2.5 \mathrm{eV}$ ) [19]. During PL test, the sample was vertically placed and the laser irradiated to the nanorods with an angle of about $60^{\circ}$. The near band emissions of PL spectra of samples A, B, 

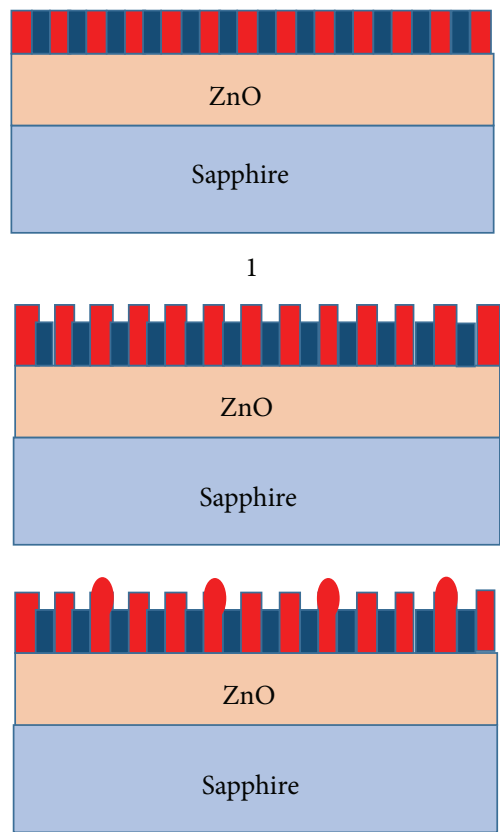

2

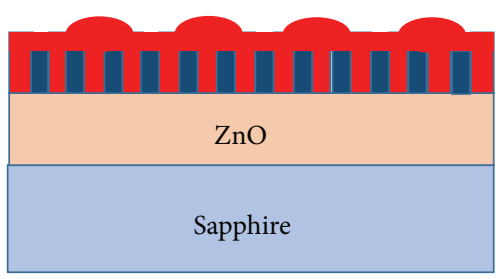

3

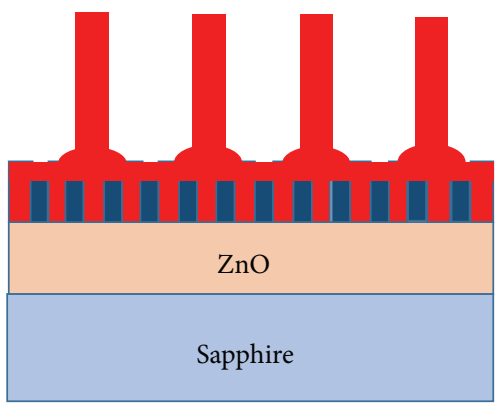

4

(a)

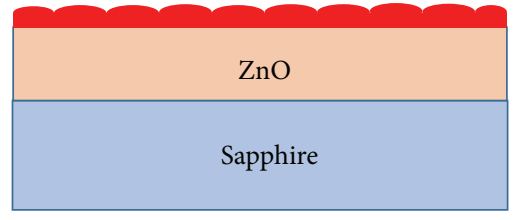

1

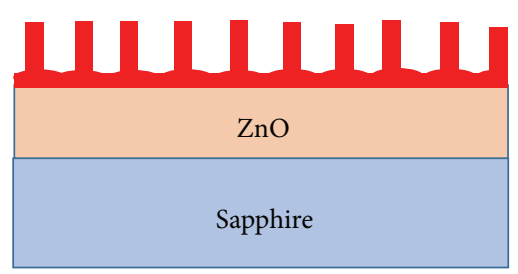

2

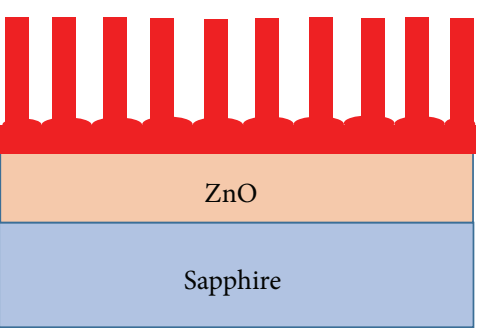

3

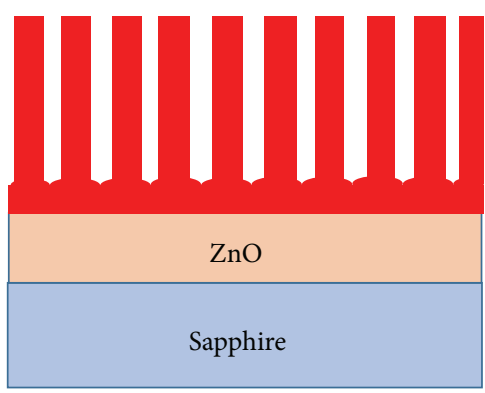

4

(b)

FIGURE 2: Growth illustrations of $\mathrm{ZnO}$ nanorods on patterned (a) and bare (b) substrates.

and $\mathrm{C}$ are shown in Figure 4(b). For sample A at the initial state of growth, small nanodisks covered the open areas on the patterned $\mathrm{SiO}_{2} / \mathrm{ZnO}$ substrate. The dominating emission located at $3.362 \mathrm{eV}$, which is due to recombination of excitons bound to donors or acceptors and similar to the observation on the patterned $\mathrm{SiO}_{2} / \mathrm{ZnO}$ substrate due to large open area of the $\mathrm{SiO}_{2} / \mathrm{ZnO}$ substrate. As the MOCVD grown $\mathrm{ZnO}$ film is generally $\mathrm{n}$-type, the peak at $3.362 \mathrm{eV}$ can be recognized as $\mathrm{D}^{0} \mathrm{X}$ [26]. Besides, a new peak at $3.368 \mathrm{eV}$ appears which can be ascribed to the surface exciton SX, which is observed in the $\mathrm{ZnO}$ films and more apparent in the $\mathrm{ZnO}$ nanostructures due to a large surface-to-volume ratio.

Compared with sample $\mathrm{A}$, more $\mathrm{ZnO}$ nanorods have been grown on sample B. It is interesting to note that a broad peak located at $3.359 \mathrm{eV}$ dominated in the spectrum, which can be easily resolved into two peaks, $3.358 \mathrm{eV}$ and $3.362 \mathrm{eV}$, respectively. The emission around $3.358 \mathrm{eV}$ has been previously ascribed as acceptor bound exciton $\left(\mathrm{A}^{0} \mathrm{X}\right)$, and the later one should be the $\mathrm{D}^{0} \mathrm{X}$ as described in sample $\mathrm{A}$. The intensity of $\mathrm{A}^{0} \mathrm{X}$ on sample $\mathrm{B}$ is much stronger than that in sample 


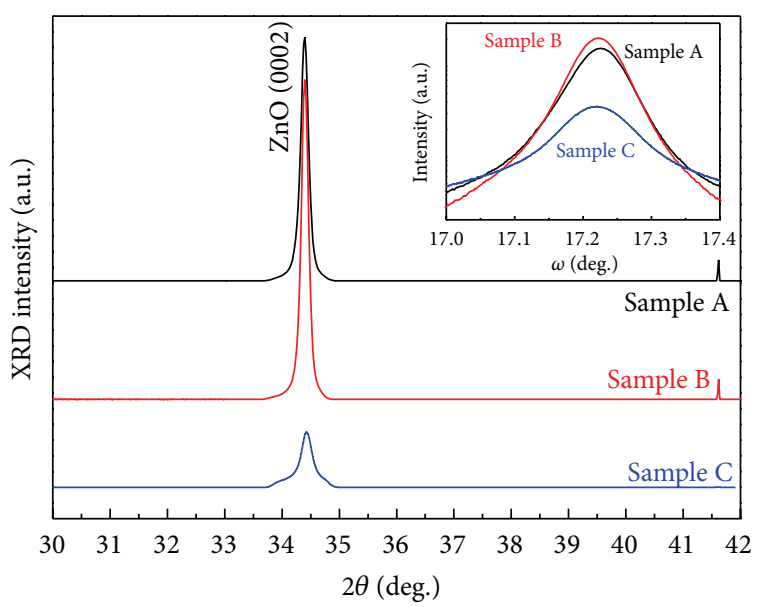

FIgURE 3: XRD patterns for all the samples. The inset shows the $\omega$ scan rocking curves.

A, where a shoulder located at $3.358 \mathrm{eV}$ can be devolved too besides the main emission peak at $3.362 \mathrm{eV}$. Similarly, the surface exciton has also been intensified compared with sample A due to more contribution from the grown nanorods. As more dense nanorods were grown on sample $\mathrm{C}$, it was expected to have a stronger $\mathrm{A}^{0} \mathrm{X}$ emission. However, opposite phenomenon is revealed as shown in Figure 4, where the main emission peak has been shifted to high energy located at $3.363 \mathrm{eV}$. Of course, a small shoulder located at $3.358 \mathrm{eV}$ can be devolved from the peak, but its intensity has become much smaller compared with sample $\mathrm{B}$. The peak-fitting result of $\mathrm{PL}$ spectrum shows that the intensity of $\mathrm{A}^{0} \mathrm{X}(3.358 \mathrm{eV})$ in sample $\mathrm{C}$ is much smaller than that of sample B. As the nanorods coverage in sample $\mathrm{C}$ is much higher, it suggested that the photoemission excited and collected from the sidewalls of the nanorods will be much smaller. The change trend of $\mathrm{A}^{0} \mathrm{X}$ emission and surface exciton SX emission for samples $\mathrm{A}, \mathrm{B}$, and $\mathrm{C}$ surely indicates that the $\mathrm{A}^{0} \mathrm{X}$ and SX should mainly originate from the sidewall surface with a small fraction comes from the top surface. The change of the $\mathrm{D}^{0} \mathrm{X}$ emission has also revealed high donor concentration contribution from the near top surface compared to the sidewall surface.

Calculation results on the formation energy of $\mathrm{Zn}$ vacancies at different positions in the slab reveal that $Z n$ vacancies are much more probably closer to the (1010) surface $(1 \mathrm{eV}$ energy lower) than inner positions in the slab [27]. This has also been supported by another report, which indicates $\mathrm{V}_{\mathrm{Zn}}$ on a nonpolar (1010) surface had significantly smaller formation energy than it did in the bulk [28]. Considering the fact that the sidewall surface is $(1010) /(10 \overline{1} 0)$ direction, the $\mathrm{A}^{0} \mathrm{X}$ emission can probably be relevant to $\mathrm{V}_{\mathrm{Zn}}$.

Figure 5 shows the green band emission of samples A, B, and $\mathrm{C}$ at temperature $9 \mathrm{~K}$. It is interesting that fine structure, which consists mainly of doublets, can be observed. The energy spacing between any two adjacent lines (LO phonon replicas) in the fine structure is about $72 \mathrm{meV}$. The number of LO-replicas that can be clearly seen is eight. At high energy side, two well-resolved series superimposing the broad band can be observed. Both of the two series equally separate fine structures and the energy spacing between them is about $27 \mathrm{meV}$. The intensity of the GB for sample C is obviously stronger than that of sample $\mathrm{B}$, which agrees well with the coverage of the $\mathrm{ZnO}$ nanorods as shown above. This indicates that the GB is mainly contributed from the $\mathrm{ZnO}$ nanorods and contribution from the $\mathrm{ZnO}$ film does not need to be taken into account here.

Raman scattering spectra of samples A, B, and C with incident light parallel to the $c$-axis of the nanorods have been shown in Figure 6. As indicated in the above discussion, the observed emissions in the PL measurements should occur from the top surface or the sidewall surface. Different to what happened in the PL measurements, the excitation laser photon energy for Raman scattering is smaller than the bandgap of $\mathrm{ZnO}$, indicating that almost scattering from both the $\mathrm{ZnO}$ nanorods and $\mathrm{ZnO}$ film can contribute to the Raman spectroscopy. In Figure 6, the observed peaks located at 99, 332, and $438 \mathrm{~cm}^{-1}$ are the classical modes of $\mathrm{ZnO}$, corresponding to $\mathrm{E}_{2}(\mathrm{low}), 2 \mathrm{E}_{2}(\mathrm{M})$, and $\mathrm{E}_{2}$ (high), respectively, which should come from both $\mathrm{ZnO}$ nanorods and films $[29,30]$. In addition to the $\mathrm{ZnO}$ modes, peaks around 276, 510,582, and $644 \mathrm{~cm}^{-1}$ are observed, with their intensity increased for samples A, B to C. The origin of these additional modes was controversial. They were assigned to $\mathrm{N}$ local vibrational modes, or $\mathrm{ZnO}$ silent modes allowed by the breakdown of the translational crystal symmetry, or $\mathrm{Zn}$ interstitial clusters due to $\mathrm{N}$ incorporation [31-33]. Regardless of the assignments, the increased intensity of the vibration modes indicates that it is contributed from the $\mathrm{ZnO}$ nanorods, not from the $\mathrm{ZnO}$ seed film. And $\mathrm{N}$ atoms have been incorporated into the $\mathrm{ZnO}$ nanorods.

The incorporation of nitrogen has been proven by XPS. Figure 7 shows the N 1s XPS line for sample C. An indistinct nitrogen signal could be seen with a high level of noise due to the low nitrogen concentration. Considering the fact that the nanorods were grown at $950^{\circ} \mathrm{C}$, at which the nitrogen solubility will drop sharply, the incorporation of nitrogen is thus low. For samples A and B, due to the looser distribution of the nanorods, it is difficult to get the nitrogen signals, while, for sample $C$, the density of the nanorods is much higher and hence a weak signal of nitrogen could be detected shown in Figure 7. It is surprising that a small amount of nitrogen can still exist in the nanorods grown at such a high substrate temperature. It is highly possible that the nitrogen atoms could be stabilized via bonding with interstitial zinc defects, forming the $\mathrm{Zn}_{\mathrm{i}}-\mathrm{N}_{\mathrm{O}}$ complexes, which has been proposed to be thermally stable. This proposal could be partially evidenced by the fact that the $\mathrm{Zn}_{\mathrm{i}}$ related Raman vibration mode at $276 \mathrm{~cm}^{-1}$ is strongest for sample $\mathrm{C}$.

As for the assignment of the acceptor, nitrogen substituting for oxygen, $\mathrm{N}_{\mathrm{O}}$, is calculated to be a deep acceptor. On the other hand, the acceptor ionization energy is predicted to be significantly reduced by the presence of various intrinsic defects that can act as efficient compensating centers and also facilitate formation of various $\mathrm{N}$-related complexes, including donor or acceptor complexes such as $\mathrm{Zn}_{\mathrm{i}}-\mathrm{N}_{\mathrm{O}}$ or $\mathrm{V}_{\mathrm{Zn}}-\mathrm{N}_{\mathrm{O}}$. In our case, we prefer to ascribe the observed acceptor to $\mathrm{V}_{\mathrm{Zn}}-\mathrm{N}_{\mathrm{O}}$ or even $\mathrm{Zn}$ vacancy clusters, or $\mathrm{Zn}$ vacancy 


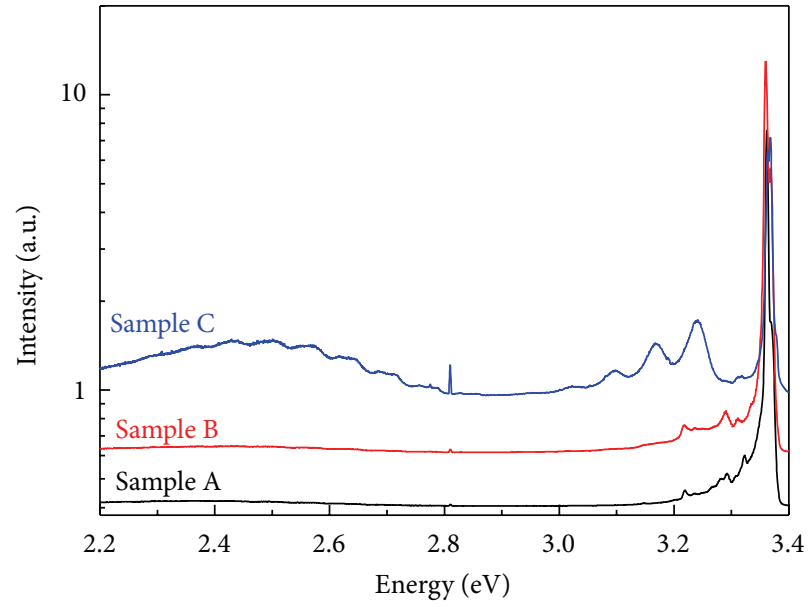

(a)

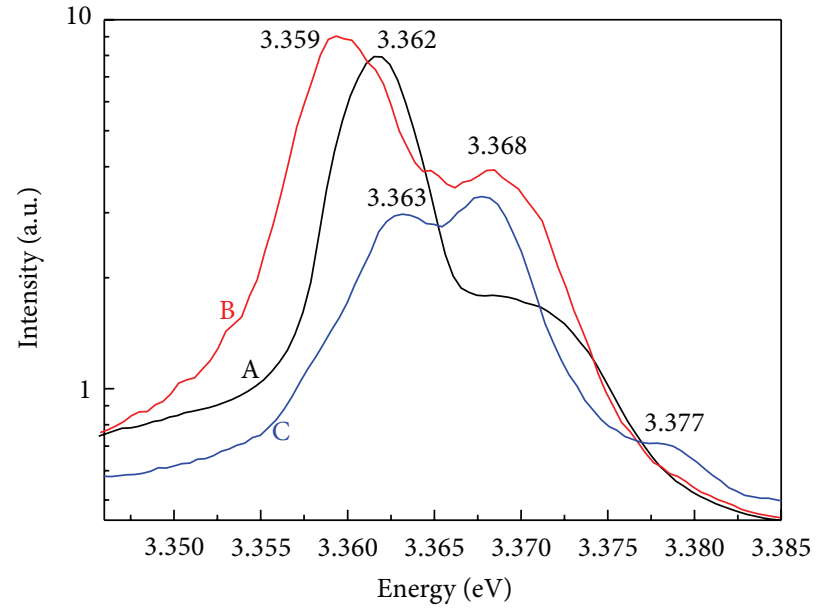

(b)

Figure 4: PL spectra of samples A, B, and C at $9 \mathrm{~K}$. (a) Full spectra from 2.2 to $3.4 \mathrm{eV}$; (b) near band edge spectra from 3.345 to $3.385 \mathrm{eV}$.

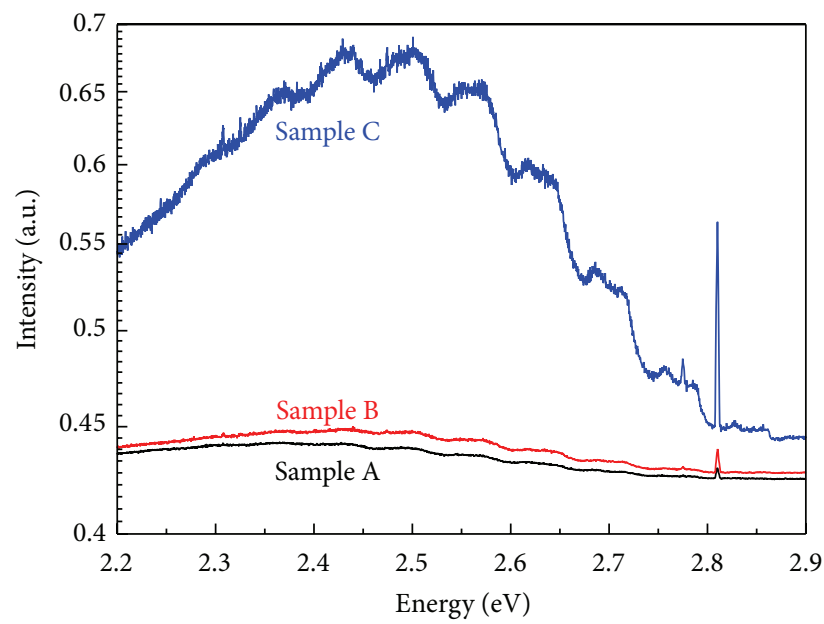

FIGURE 5: Green band emission of PL spectra of samples A, B, and C.

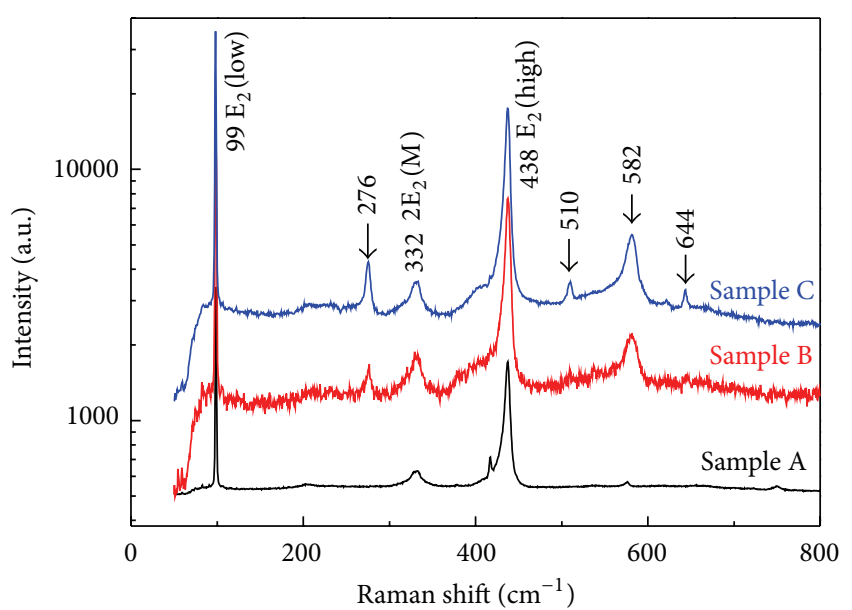

FIGURE 6: Raman scattering spectra of samples A, B, and C.

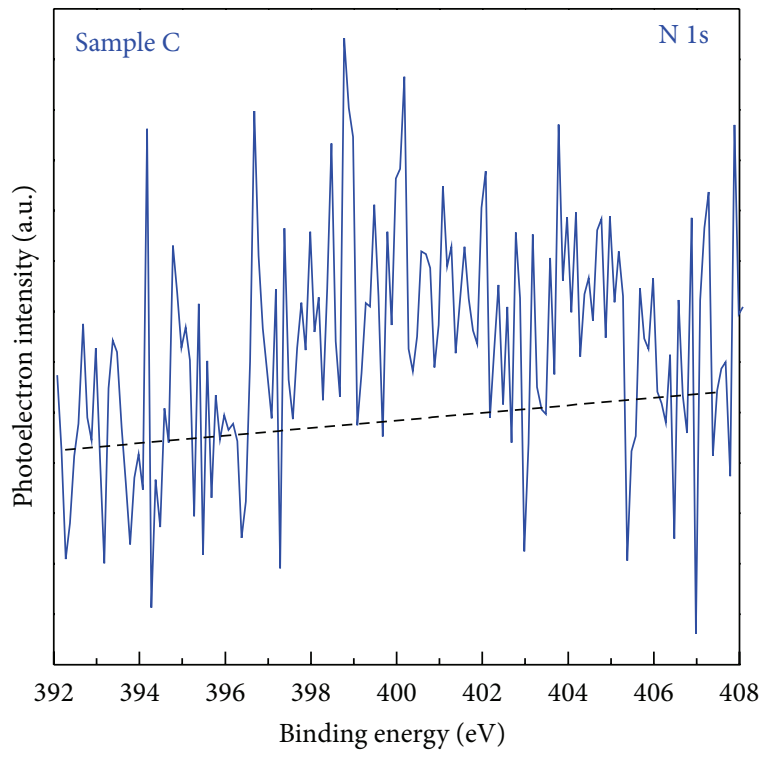

FIgURE 7: Nitrogen 1s XPS line of sample C.

related complex should be a better description of the shallow acceptor. These acceptors are believed to locate near the sidewall surface according to the enhanced $\mathrm{A}^{0} \mathrm{X}$ emission observed in sample B, possibly due to the modification of large surface-to-volume ratio on the formation energy of the acceptors.

\section{Conclusions}

The paper illustrates different growth process of $\mathrm{ZnO}$ nanorods on patterned and bare $\mathrm{ZnO}$ substrates. $\mathrm{ZnO}$ nanorods of different intensity and different surface-tovolume ratio have been grown. From the PL test, we can see that the defects in $\mathrm{ZnO}$ nanorods are not uniformly distributed in the slab. The $\mathrm{A}^{0} \mathrm{X}$ emission related defects, which 
may be relevant to $\mathrm{V}_{\mathrm{Zn}}$, are more likely to distribute in the sidewall surface, while $\mathrm{D}^{0} \mathrm{X}$ related defects uniformly distribute in the near top surface. The Raman spectra ensure the fact that $\mathrm{N}$ atoms have been incorporated into the $\mathrm{ZnO}$ nanorods. Our work infers how to realize the modification on the formation energy of the acceptor, like the effect of the large surface-to-volume ratio, which is critical for the realization of the p-type doping in $\mathrm{ZnO}$ films.

\section{Conflict of Interests}

The authors declare that there is no conflict of interests regarding the publication of this paper.

\section{Acknowledgments}

This research was supported by the State Key Program for Basic Research of China under Grant no. 2011CB302003, National Natural Science Foundation of China (nos. 6103020, 60990312, 61274058, and 61322403), Basic Research Program of Jiangsu Province (BK20130013), and the Priority Academic Program Development of Jiangsu Higher Education Institutions.

\section{References}

[1] J. B. K. Law and J. T. L. Thong, "Simple fabrication of a $\mathrm{ZnO}$ nanowire photodetector with a fast photoresponse time," Applied Physics Letters, vol. 88, no. 13, Article ID 133114, 2006.

[2] M. S. Arnold, P. Avouris, Z. W. Pan, and Z. L. Wang, "Field-effect transistors based on single semiconducting oxide nanobelts," Journal of Physical Chemistry B, vol. 107, no. 3, pp. 659-663, 2003.

[3] R. Könenkamp, R. C. Word, and C. Schlegel, "Vertical nanowire light-emitting diode," Applied Physics Letters, vol. 85, no. 24, pp. 6004-6006, 2004.

[4] H. T. Ng, J. Han, T. Yamada, P. Nguyen, Y. P. Chen, and M. Meyyappan, "Single crystal nanowire vertical surround-gate field-effect transistor," Nano Letters, vol. 4, no. 7, pp. 1247-1252, 2004.

[5] M. Law, L. E. Greene, J. C. Johnson, R. Saykally, and P. D. Yang, "Nanowire dye-sensitized solar cells," Nature Materials, vol. 4, pp. 455-459, 2005.

[6] J. Y. Park, Y. S. Yun, Y. S. Hong, H. Oh, J.-J. Kim, and S. S. Kim, "Synthesis, electrical and photoresponse properties of vertically well-aligned and epitaxial $\mathrm{ZnO}$ nanorods on $\mathrm{GaN}$ buffered sapphire substrates," Applied Physics Letters, vol. 87, no. 12, Article ID 123108, pp. 1-3, 2005.

[7] Y. Zhang, H. Jia, R. Wang et al., "Low-temperature growth and Raman scattering study of vertically aligned $\mathrm{ZnO}$ nanowires on Si substrate," Applied Physics Letters, vol. 83, no. 22, pp. 46314633, 2003.

[8] C. H. Wang, A. S. W. Wong, and G. W. Ho, "Facile solution route to vertically aligned, selective growth of $\mathrm{ZnO}$ nanostructure arrays," Langmuir, vol. 23, no. 24, pp. 11960-11963, 2007.

[9] Y. Tak and K. Yong, "Controlled growth of well-aligned $\mathrm{ZnO}$ nanorod array using a novel solution method," Journal of Physical Chemistry B, vol. 109, no. 41, pp. 19263-19269, 2005.

[10] Y.-J. Kim, C.-H. Lee, Y. J. Hong, G.-C. Yi, S. S. Kim, and H. Cheong, "Controlled selective growth of $\mathrm{ZnO}$ nanorod and microrod arrays on Si substrates by a wet chemical method," Applied Physics Letters, vol. 89, no. 16, Article ID 163128, 2006.

[11] Q. Ahsanulhaqa, S. H. Kim, and Y. B. Hahn, "Hexagonally patterned selective growth of well-aligned $\mathrm{ZnO}$ nanorod arrays," Journal of Alloys and Compounds, vol. 484, pp. 17-20, 2009.

[12] H. Wang, Z. P. Zhang, X. N. Wang et al., "Selective growth of vertical-aligned $\mathrm{ZnO}$ nanorod arrays on si substrate by catalystfree thermal evaporation," Nanoscale Research Letters, vol. 3, no. 9, pp. 309-314, 2008.

[13] A. Qurashi, J. H. Kim, and Y. B. Hahn, "Density-controlled selective growth of well-aligned $\mathrm{ZnO}$ nanorod arrays by a hybrid approach," Superlattices and Microstructures, vol. 48, no. 2, pp. 162-169, 2010.

[14] L. Ressier, E. Palleau, and S. Behar, "Electrical nano-imprint lithography," Nanotechnology, vol. 23, no. 25, Article ID 255302, 2012.

[15] K.-B. Choi and J. J. Lee, "Passive compliant wafer stage for single-step nano-imprint lithography," Review of Scientific Instruments, vol. 76, no. 7, Article ID 075106, 2005.

[16] B. Meyer and D. Marx, "Density-functional study of the structure and stability of $\mathrm{ZnO}$ surfaces," Physical Review B, vol. 67, Article ID 035403, 2003.

[17] D. C. Oh, T. Kato, H. Goto et al., "Comparative study of photoluminescences for $\mathrm{Zn}$-polar and O-polar faces of singlecrystalline ZnO bulks," Applied Physics Letters, vol. 93, no. 24, Article ID 241907, 2008.

[18] M. W. Allen, P. Miller, R. J. Reeves, and S. M. Durbin, "Influence of spontaneous polarization on the electrical and optical properties of bulk, single crystal ZnO," Applied Physics Letters, vol. 90, no. 6, Article ID 062104, 2007.

[19] A. Yamamoto, Y. Moriwaki, K. Hattori, and H. Yanagi, "A comparative study of photoluminescence of $\mathrm{Zn}$-polar and O-polar faces in single crystal $\mathrm{ZnO}$ using moment analysis," Applied Physics Letters, vol. 98, no. 6, Article ID 061907, 2011.

[20] V. Consonni, E. Sarigiannidou, E. Appert et al., "Selective area growth of well-ordered $\mathrm{ZnO}$ nanowire arrays with controllable polarity," ACS Nano, vol. 8, no. 5, pp. 4761-4770, 2014.

[21] C. Klingshirn, J. Fallert, H. Zhou et al., "65 years of $\mathrm{ZnO}$ research-old and very recent results," Physica Status Solidi B, vol. 247, no. 6, pp. 1424-1447, 2010.

[22] A. B. Djuriić, A. M. C. Ng, and X. Y. Chen, "ZnO nanostructures for optoelectronics: material properties and device applications," Progress in Quantum Electronics, vol. 34, no. 4, pp. 191-259, 2010.

[23] V. Khranovskyy, V. Lazorenko, G. Lashkarev, and R. Yakimova, "Luminescence anisotropy of $\mathrm{ZnO}$ microrods," Journal of Luminescence, vol. 132, no. 10, pp. 2643-2647, 2012.

[24] S. S. Kurbanov, H. D. Cho, and T. W. Kang, "Effect of excitation and detection angles on photoluminescence spectrum from $\mathrm{ZnO}$ nanorod array," Optics Communications, vol. 284, no. 1, pp. 240-244, 2011.

[25] H. Chen, S. Gu, K. Tang et al., "Origins of green band emission in high-temperature annealed $\mathrm{N}$-doped $\mathrm{ZnO}$," Journal of Luminescence, vol. 131, no. 6, pp. 1189-1192, 2011.

[26] J. Xu, R. Ott, A. S. Sabau et al., "Generation of nitrogen acceptors in $\mathrm{ZnO}$ using pulse thermal processing," Applied Physics Letters, vol. 92, no. 15, Article ID 151112, 2008.

[27] F. Fabbri, M. Villani, A. Catellani et al., “Zn vacancy induced green luminescence on non-polar surfaces in $\mathrm{ZnO}$ nanostructures," Scientific Reports, vol. 4, article 5158, 2014. 
[28] K. Chae, Y. C. Chung, and H. Kim, "Ferromagnetism induced by vacancies in bulk and the (1010) surfaces of $\mathrm{ZnO}$ : density functional theory calculations," Japanese Journal of Applied Physics, vol. 50, Article ID 01BE05, 2011.

[29] R. H. Callender, S. S. Sussman, M. Selders, and R. K. Chang, "Dispersion of Raman cross section in $\mathrm{CdS}$ and $\mathrm{ZnO}$ over a wide energy range," Physical Review B, vol. 7, no. 8, pp. 3788-3798, 1973.

[30] F. Decremps, J. Pellicer-Porres, A. M. Saitta, J.-C. Chervin, and A. Polian, "High-pressure Raman spectroscopy study of wurtzite ZnO," Physical Review B, vol. 65, no. 9, Article ID 092101, 4 pages, 2002.

[31] A. Kaschner, U. Haboeck, M. Strassburg et al., "Nitrogen-related local vibrational modes in ZnO:N," Applied Physics Letters, vol. 80, no. 11, pp. 1909-1911, 2002.

[32] X. Zhu, H.-Z. Wu, D.-J. Qiu et al., "Photoluminescence and resonant Raman scattering in $\mathrm{N}$-doped $\mathrm{ZnO}$ thin films," Optics Communications, vol. 283, no. 13, pp. 2695-2699, 2010.

[33] S. J. Jokela and M. D. McCluskey, "Unambiguous identification of nitrogen-hydrogen complexes in ZnO," Physical Review B, vol. 76, Article ID 193201, 2007. 

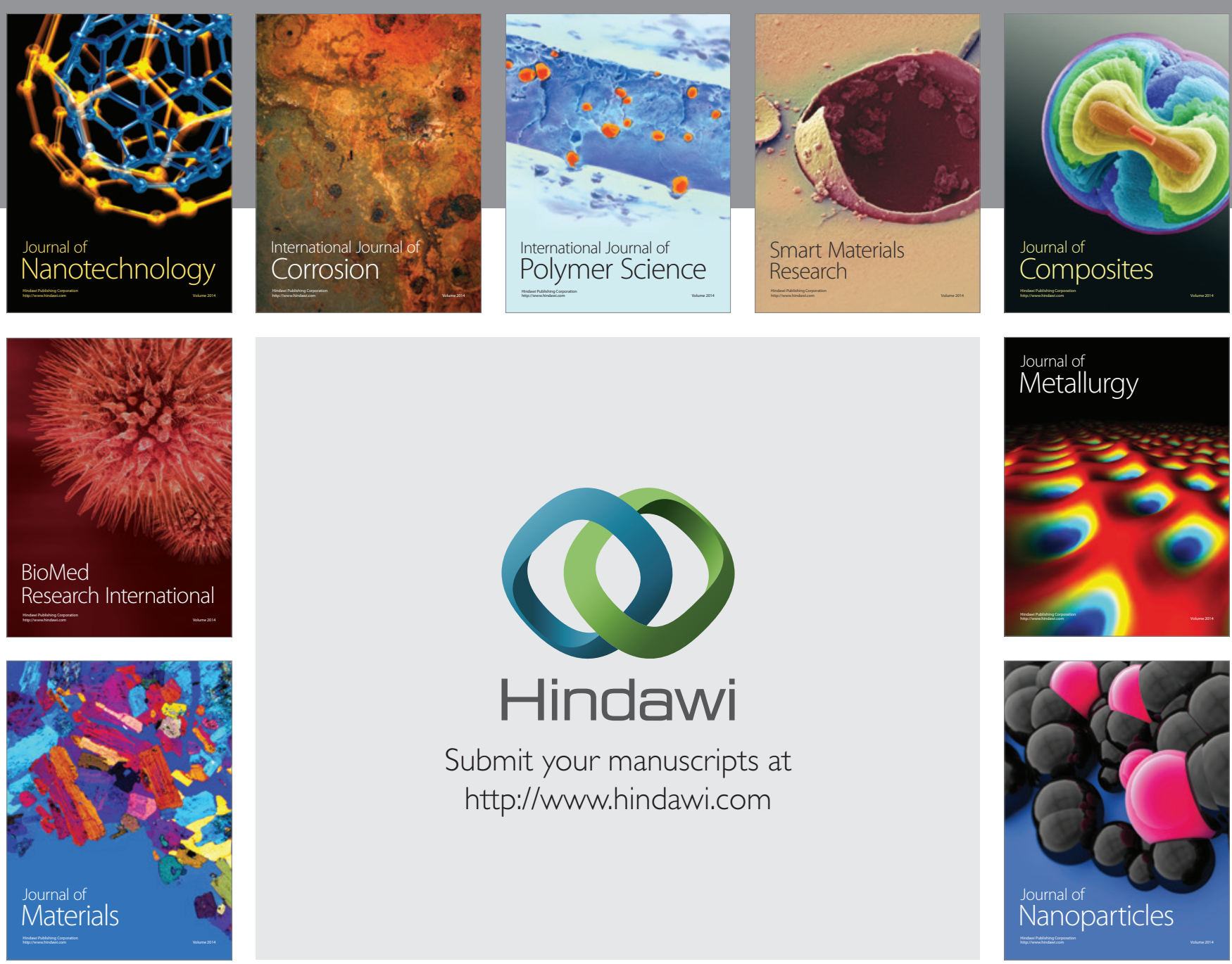

Submit your manuscripts at http://www.hindawi.com
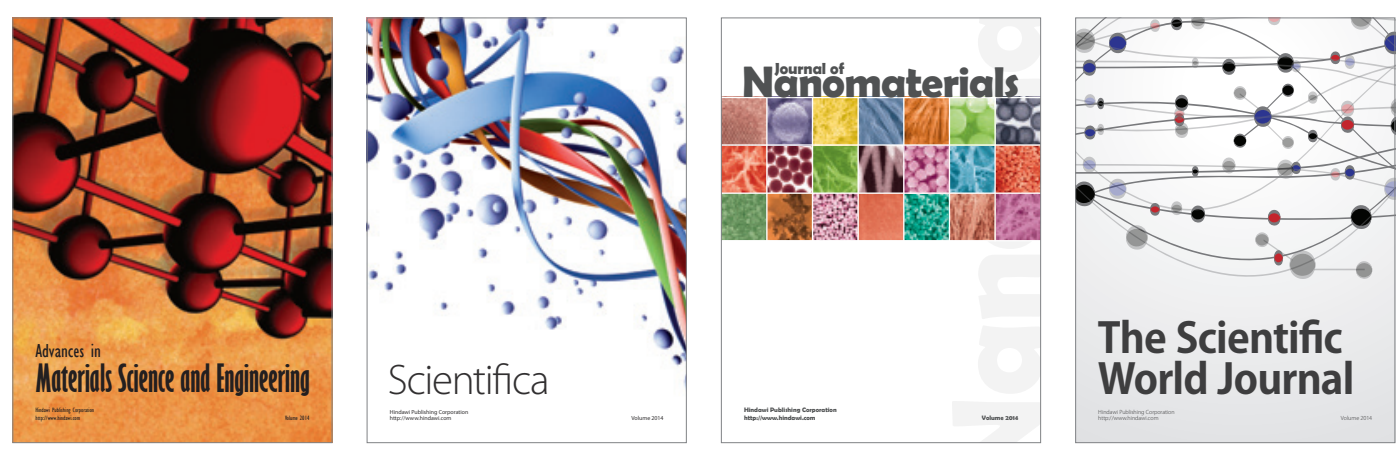

\section{The Scientific World Journal}
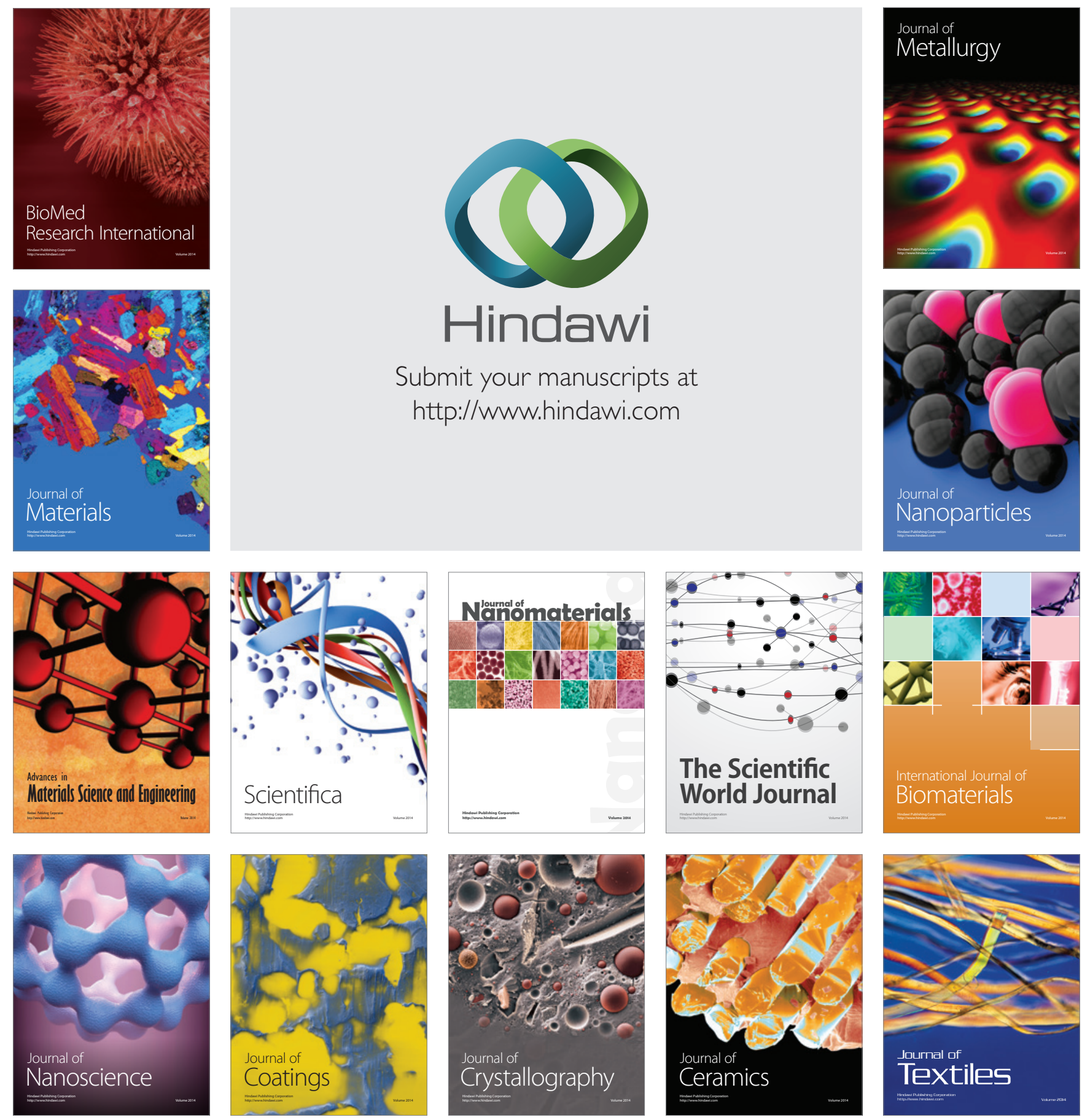